\title{
Base + Add-on Pricing in Extremely Competitive Categories
}

\author{
Ruijiao Guo $^{1} \cdot$ Purushottam Papatla ${ }^{1}$
}

Published online: 29 April 2015

(C) Springer Science+Business Media New York 2015

\begin{abstract}
Firms have typically relied on the base + add-on pricing model to stimulate ratings in extremely competitive categories like mobile applications. In most cases, the base is the basic version of the product and is offered for free or at a very low price. Product complements or additional product features serve as the add-ons. The standing assumption seems to be that the low base price encourages acquisition and use of the product followed by purchase of the add-ons. Eventually, this should increase both the valence and volume of ratings posted by consumers and, hence, lead to higher sales. The relationship between prices, valence, and volume of ratings, however, is yet to be formally tested in the literature. This is the focus of our research. Specifically, we investigate the relationships between various combinations of base and add-on prices and the valence and volume of ratings. Our investigation is based on a conceptual framework in which prices play both informative and allocative roles and are also maintained in mental accounts by consumers. The framework leads us to six research questions which we investigate empirically in a setting of over 9,000 mobile apps. Our results suggest that a combination of low base and high add-on prices will lead to high valence and volume of ratings. Interestingly, we find that the widely used combination of low base and low
\end{abstract}

Purushottam Papatla

papatla@uwm.edu

Ruijiao Guo

rguo@uwm.edu

1 University of Wisconsin-Milwaukee, Milwaukee, WI, USA add-on prices will result in low valence and volume of ratings. This pricing option is, therefore, not attractive for firms. Our key finding, therefore, is that the classic low base + high addon pricing model is the best option to generate high volumes of positive word of mouth from consumers. Firms, therefore, should not rely on lowering both base and add-on prices to stimulate consumer ratings even in extremely competitive categories. We also develop additional pricing implications of our findings for products in extremely competitive categories via a simulation of the effects of switching between price combinations.

Keywords Extremely competitive categories $\cdot$ Base + add-on pricing - Consumer ratings - Valence and volume of ratings . Mobile apps

\section{Introduction}

Many categories today are extremely competitive for sellers. For instance, the two large distributors in the mobile app category—Google's Play store and Apple's App Store—offered more than two million products as of April 2014. Similarly, more than 150,000 software products from download.com and more than 40,000 from tucows.com can be obtained for free or for a small price. Online music and radio services (e.g., spotify.com and pandora.com) are additional examples of extremely competitive categories.

The plethora of choices increases consumers' reliance on user ratings and product popularity [14] while choosing products. For instance, Dellarocas et al [6] find that the valence of user ratings affects the box-office sales of movies. Interestingly, popularity itself is inferred from the number of ratings that a product receives [35]. Thus, valence as well as the volume of ratings could affect whether consumers are willing to try a 
product. Businesses in extremely competitive categories, therefore, need to actively manage consumer opinions and take steps to increase both.

Firms have typically relied on the base + add-on pricing $\operatorname{model}^{1}[20,30]$ to stimulate ratings in extremely competitive categories. In most cases, the base is the basic version of the product and is offered for free or at a very low price. Product complements or additional product features serve as the addons. The standing assumption seems to be that the low base price encourages acquisition and the use of the product and will be followed by purchase of the add-ons. Eventually, this should lead to an increase in both the valence and volume of ratings posted by consumers and, hence, lead to higher sales. The relationship between prices and the valence and volume of ratings, however, is yet to be formally tested and several questions remain unanswered. For instance, is the classic combination of low base price and high add-on price the most effective in increasing the valence and volume of ratings or is it better to price both base and add-ons low? Alternatively, is a combination of a high base price and low add-on prices likely to result in high valence and volume of ratings? Our goal is to address such questions through findings that can provide insights on how firms should price base products and add-ons to increase the valence and volume of user ratings in extremely competitive categories.

Our investigation is based on a conceptual framework in which prices play both informative and allocative roles [23] and are also maintained in mental accounts [28] by consumers. The framework leads us to six research questions which we investigate empirically in a setting of over 9,000 mobile apps. This is a relevant category to investigate for multiple reasons. First, the two largest distributors, Google's Play Store and Apple's App Store, regularly solicit user opinions and present summaries in the form of valence and volume of consumer ratings of apps to shoppers. User acquisition in the category is, therefore, likely to be affected by consumer ratings. Second, most app firms rely on the base + add-on pricing model and sales of in-app features for revenues [10]. Third, firms vary substantially in the combination of prices that they use. In the sample of over 9,000 apps that we investigate, for instance, base prices varied from $\$ 0$ to $\$ 20$ and the average price of add-on features ranged between $\$ 0$ and $\$ 999$. Our sample, therefore, demonstrates the variability in prices that permits us to investigate the relationships between various combinations of prices and the valence and volume of ratings.

Finally, and importantly, ratings do appear to vary substantially with base and add-on prices. For instance, an app titled Pixel Gun 3D in our sample is offered for free - a base price of $\$ 0$ - but the add-on features have an average price of $\$ 19.79$ which is substantially higher than the average of $\$ 8.91$ for all

${ }^{1}$ Tripsas and Gavetti [30] refer to this as the "razor/blade business model" (p. 1148). the other products in our sample. The product, therefore, follows a low base and high add-on prices model. The volume of ratings for this product was 13,984 and the average valence was 4.8 out of a possible 5 . On the other hand, a different product in the sample, Namco Arcade, relied on the practice widely used in the mobile app category of a free base price and low add-on prices which averaged $\$ 2.39$. This product, however, received only 754 ratings with an average valence of 2.8 over almost 2 years.

Next, we review the literature and develop our conceptual framework. We follow this with a section that motivates and describes our research questions. We then describe our data and the empirical model for our investigation. Following this, we present our results and the results of a simulation analysis of how the valence and volume of ratings vary with a change in the base and add-on price combinations. Finally, we discuss the implications of our findings for the base + add-on pricing model in extremely competitive categories and provide directions for additional research.

\section{Overview of the Literature}

\subsection{Informative and Allocative Roles of Price}

Economists [13] and marketing scholars [23] have long reported that price has dual roles in consumer decision-making. First, it plays an informative role since consumers often infer product quality $[17,24]$ and also form performance expectations [8] based on observed prices. The reliance on price to assess a product's quality or performance is particularly likely in extremely competitive categories [5]. Second, price also plays an allocative role due to budget constraints $[4,12]$ faced by consumers who need to allocate available monetary resources across multiple products. Thus, as the price of a product increases, less would be available to allocate to other products [9] if the product is purchased.

\subsection{Effects of the Informative Role of Price on Ratings}

In categories where most firms set prices that are close to zero, prices that are not very low may signal better quality or features in a product. In fact, higher prices in such categories could be "thought provoking and enhance the perception of relevance" thus increasing the likelihood of choice ([32], p.118) and, hence, the incidence of consumer ratings. Thus, higher prices of the base, add-on, or both can affect the volume of ratings in extremely competitive categories.

The informative role of price not only affects volume but also the valence of consumer ratings. Two mechanisms contribute to this effect. First, the formation of performance expectations results in consumer judgments of products, postpurchase, based on those expectations. The literature $[8,21$, 
22, 31] provides ample evidence for this. Second, findings also suggest that consumers who pay a higher price for a product bolster their judgments of its performance [19] to minimize cognitive dissonance. Both mechanisms should therefore lead to a relationship between base and add-on prices and the valence of consumer ratings.

\subsection{Effect of the Allocative Role of Price on Ratings}

The price paid for a product also affects its perceived value which is the "consumer's overall assessment of the utility of a product based on perceptions of what is received and what is given" ([34], p.14). Perceived value is important because it can affect whether consumers choose the product and, even if they do, how satisfied they are with it. High prices can reduce perceived value, lower the likelihood of choice, and hence, the volume of ratings.

Consumers also maintain mental accounts of their spending on different categories of products as well as individual products and activities $[16,28]$. In the context of base + addon pricing, the maintenance of product-specific mental accounts means that an increase in the base price or the prices of the add-ons) increases the monetary allocation to the product and reduces its perceived value. Changes in perceived value can affect consumers' satisfaction with products [26] with higher perceived value leading to greater satisfaction and vice versa [11]. Multiple studies have demonstrated this effect across a variety of categories [26] and in online settings as well [33].

Customers who are highly satisfied or dissatisfied are also likely to engage in more word of mouth. Those who are highly satisfied may discuss the product with others to be altruistic or to appear well-informed [3,7]. Those who are dissatisfied, on the other hand, do so to reduce their stress, out of altruism (so that others don't have the same poor experience), or as a means of getting back at the firm that sold them the product [25]. Anderson [1] finds empirical evidence for such effects of satisfaction on the volume of word of mouth and reports that both high and low satisfaction increase volume. Satisfaction also affects the valence of consumer ratings [18] with higher (lower) satisfaction leading to higher (lower) valence. Thus, in the allocative role as well, base and add-on prices affect the valence and volume of ratings.

In addition to their main effects, base and add-on prices can have interactive effects as well on consumer ratings. Thus, for instance, a low base price may increase the volume or valence of ratings due to high perceived value. As the number of purchased add-ons increases, and the total allocation to the product rises, however, perceived value and satisfaction may come down and higher volumes of lower valence ratings may result.

\subsection{Conceptual Framework}

Figure 1 illustrates our conceptual framework of how the informative and allocative roles of base and add-on prices affect the volume and valence of consumer ratings in extremely competitive categories. The effects of the informative role flow through paths that can be traced by following segments 1,2 , or 3 , from the left to the right in the figure. The path connected by segments marked 1 relates to the role of prices in influencing perceptions of quality and, hence, the number of consumers who select the product over competing products. The larger the number of consumers who choose the product, the more would be the volume of ratings that it receives and vice versa. The path along segments numbered 2 traces the effects of pre-purchase expectations set by prices which influence post-purchase evaluations and consumer satisfaction. Satisfaction is also affected by the bolstering effect of prices on post-purchase evaluations through the path connected by segments numbered 3. Eventually, satisfaction affects both volume and valence of ratings.

The allocative role of prices affects perceived value and, hence, both choice and satisfaction. An increase in the total of the two prices assigned to the account reduces perceived value and vice versa. Changes in perceived value affect choice and, hence, the volume of ratings through the path with segments numbered 4. Variations in perceived value also affect satisfaction which then affects volume and valence through segments numbered 5.

\section{Research Questions}

The base + add-on pricing model is widely used in the mobileapp category. Most firms give their products away for free or sell them at a very low base price. Additionally, firms obtain much of their revenues through sales of what the industry refers to as in-app products. For instance, the popular app Candy Crush Saga obtains most of its revenue from sales of in-app products called Boosters. We, therefore, use the mobile app category as the research setting for our empirical investigation. Accordingly, each of our research questions relates to the effects of the base price, the prices of the in-app products, and the interaction of the two on the valence and volume of consumer ratings for apps. The first three questions are regarding the effects of prices on the valence of ratings and the next three are on how prices affect the volume of ratings.

\subsection{Question 1: Does the Base Price Affect the Valence of Ratings?}

Our first question is on the effect of the base price of an app on the valence of ratings and follows from the informative and allocative effects of price. Three paths formed by segments 
Conceptual Framework

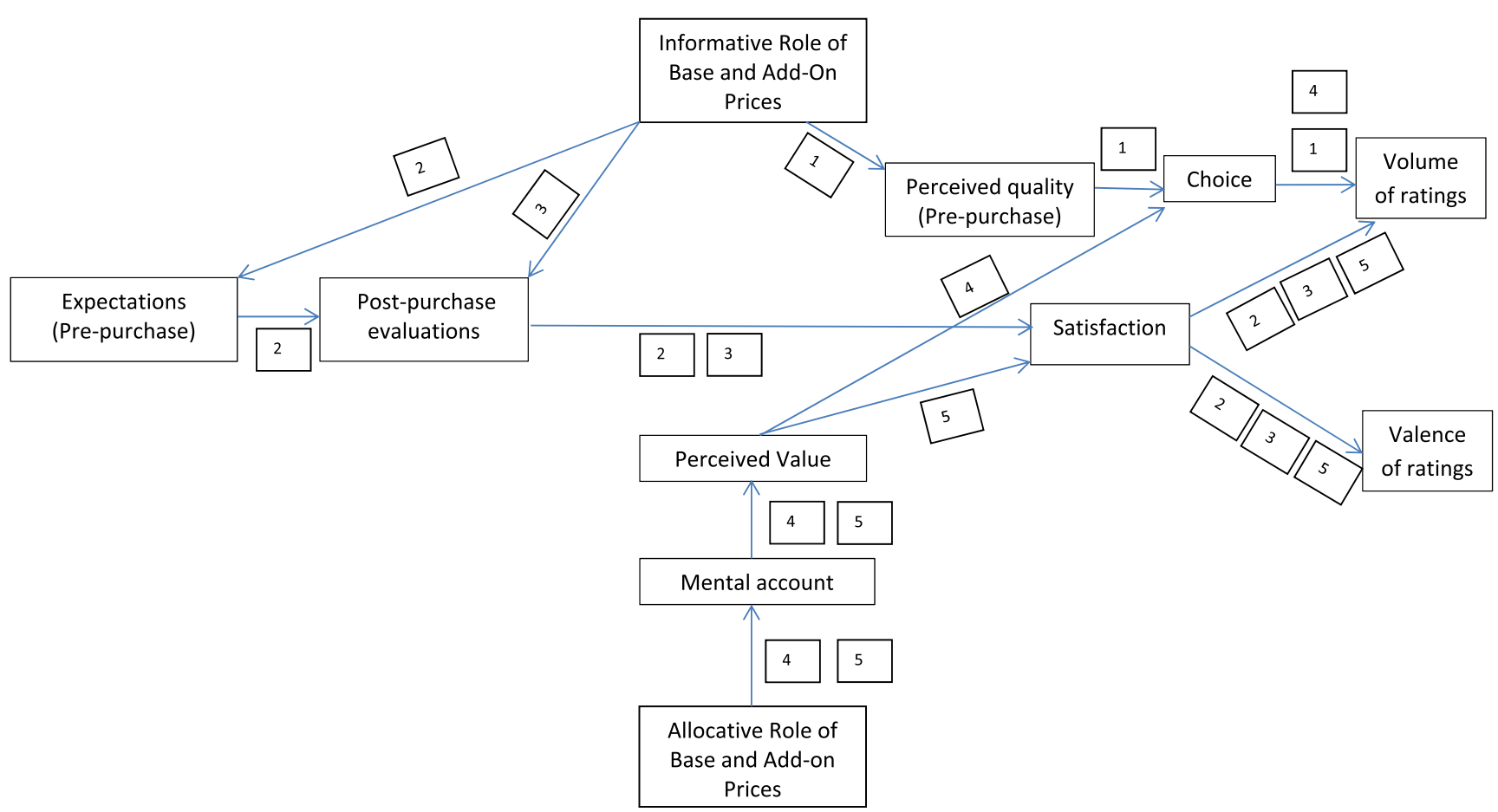

Fig. 1 Conceptual framework

numbered 2, 3, and 5 suggest that base prices can affect valence. If an app exceeds (falls below) expectations, a high base price will lead to higher (lower) ratings. Whether the valence of ratings has a positive or negative relationship with base price, therefore, depends on how the product meets expectations. The effects of bolstering (19), however, should lead to higher ratings as price increases. This means that valence should have a positive relationship with base price. Finally, an increase (decrease) in the base price leads to a reduction (increase) in perceived value and satisfaction and corresponding changes in the valence of ratings.

\subsection{Question 2: Do In-App Prices Affect the Valence of Ratings?}

In-app prices also affect valence through their informative and allocative roles. Large distributors of mobile apps like the App Store and Play Store display the range of the in-app prices of each app. These prices, therefore, should also affect valence through paths with segments numbered 2 and 3 . Thus, higher (lower) in-app prices should raise (lower) pre-purchase expectations and affect valence based on how the product meets the expectations. Additionally, higher in-app prices should lead to product bolstering and affect post-purchase evaluations.

Most apps can be used without buying additional features. In-app features are, therefore, likely to be purchased only by those who use the app, are satisfied with it, and desire to obtain additional utility. Since receipt of additional utility increases value, in-app prices should have a positive effect on valence despite increasing the monetary resources allocated to the app's mental account. This effect flows through the path with segments numbered 5 .

\subsection{Question 3: Do the Base Price and In-App Prices Have an Interactive Effect in the Valence of Ratings?}

Mental accounting means that the perceived value from the same amount spent on in-app purchases comes down as base prices increase. Thus, for instance, a dollar spent on an in-app feature for an app that is free could be perceived as giving more value than the same amount spent on an in-app feature for an app whose base price is four dollars. Hence, base price and in-app prices should have a negative interactive effect on the valence of ratings. This interactive effect flows through the path with segments numbered 5 .

\subsection{Question 4: Does the Base Price Affect the Volume of Ratings?}

A low (high) price should reduce (increase) perceived quality and have corresponding effects on choice and the volume of ratings. These effects flow through the path with segments marked 1. A low base price should also lower consumer expectations regarding the product. It should, therefore, reduce the potential for dissatisfaction and, hence, the volume of ratings. It should also lower the consumer's need to reduce 
cognitive dissonance through product bolstering. Low base prices should, therefore, reduce consumers' desire to discuss the product with others and, hence, reduce the volume of ratings. On the other hand, a high base price should increase volume. Specifically, consumer expectations and the need to reduce cognitive dissonance should be higher. In light of this, if the product performs below expectations, consumers should be dissatisfied and should be more likely to announce their experience to others $[1,25]$. Interestingly, even if the product exceeds expectations and results in increased satisfaction, consumers should be more interested in sharing their experience with others [3, 7]. Likewise, the need for product bolstering should also increase as price increases and, hence, increase perceived performance, satisfaction, and the interest in promoting the product to others. Overall, therefore, low (high) base prices should result in low (high) volume of ratings through paths with segments numbered 2 and 3.

A low (high) base price can increase (decrease) perceived value and, hence, increase (decrease) the number of consumers who choose the product. The number of consumers choosing the product affects the incidence of choice and, hence, the volume of ratings. On the other hand, both increase and decrease in satisfaction should increase the volume of ratings. The effects due to choice and satisfaction on volume flow through the paths with segments marked 4 (for choice) and 5 (for satisfaction). Given the very different effects, however, whether base prices increase or decrease the volume of ratings is an empirical outcome.

\subsection{Question 5: Do In-App Prices Affect the Volume of Ratings?}

In-app prices affect the volume of ratings in their informative and allocative roles. In the informative role, as discussed previously, the prices of in-app features displayed by distributors to prospective customers affect perceived quality and, hence, choice and the volume of ratings through the path with segments marked 1 . The displayed in-app prices also affect the volume of ratings in their allocative role by influencing perceived value and choice (path with segments numbered 4). Higher (lower) in-app prices reduce (increase) perceived value and, hence, choice and volume of ratings. On the other hand, in-app features are likely to be bought by users who use the app and seek additional utility. Thus, despite the allocation of the purchase amounts of in-app features to the app's mental account, the increase in utility should increase the app's value thus increasing satisfaction and the volume of ratings (through the paths with segments marked 5). Thus, higher in-app prices can either increase or decrease the volume of ratings through various paths. Their net effect, therefore, is also an empirical outcome.

\subsection{Question 6: Do the Base Price and In-App Prices Have an Interactive Effect in the Volume of Ratings?}

An increase in base prices and in-app prices increases the amount allocated to an app's mental account thus reducing its value and, hence, the consumer's satisfaction with it (path with segments marked 5). This should increase the volume of ratings. A decrease in base prices and in-app prices should also increase volume since it reduces the amount allocated to the app's mental account thus increasing value and satisfaction. Overall, therefore, base prices and in-app prices should have a positive interactive effect on the volume of ratings.

\section{Research Setting and Variables}

We use data from the mobile app category for our empirical investigation. Most apps in the market are developed for Google's Android operating system, Apple's iOS, or both, and are distributed through large distributors like Google Play and App Store. Further, the number of apps carried by each store is very high. The App Store alone, for instance, had more than 1 million apps by the end of 2013 and 25 to 30 thousand new apps are added each month [10]. The mobile app category is therefore extremely competitive and serves as an appropriate setting for our research.

Distributors present summaries of valence and volume of the ratings of each app to potential users. The displays of each app also include product details such as size, the launch-date and the price. Each app is also given space on the page to include several screenshots to explain its features. Additionally, distributors may promote some apps and present them to visitors either as soon as they arrive at the store or as they are browsing through different categories of apps.

We collected our data on April 29, 2014 on all the apps available in the 18 categories of games such as action, adventure, and arcade, for Apple's iOS devices from the site App Annie. ${ }^{2}$ Of the 10,772 apps that were available on that date, 1,739 apps did not have data on at least some of the variables that we consider in our analysis. We, therefore, dropped them from our sample and retained the remaining 9,033 apps for our investigation. Some of these apps can be used with multiple devices such as the iPod, iPad, and Mac. For each app, we collected all of the information provided by the App Store including whether it was promoted by the store. Additionally, we collected data on the number of languages that the app is available in, the number of versions that it was released in, and its size. We also collected data on the number and pricing of in-app features of each app. The number of apps offered by each firm was also collected to control for heterogeneity of firms, for instance, in their ability to stimulate consumer ratings.

\footnotetext{
${ }^{2}$ https://www.appannie.com/apps/ios/top/united-states/ games/action/.
} 


\subsection{Dependent Variable(s)}

Our two dependent variables are the Valence and Volume of ratings of each app. The mean valence was above 4 and typically below the median and mode for each of the 18 categories, on a rating scale of $1-5,5$ being high. It was, thus, leftskewed. On the other hand, the mean volume of ratings was substantially higher than the median and mode and, hence, right-skewed for most categories. We, therefore, logtransform and standardize both variables.

\subsection{Independent Variables}

The independent variables of primary interest for our investigation are the Price of the App and the prices of the in-app features. Since an app may have multiple in-app features at various prices, we use a number of variables to represent inapp features and prices. Specifically, we include a variable for the Number of In-App Features offered by the app. Additionally, we obtain the Mean In-App Price, Minimum In-App Price, and the Standard Deviation of In-App Prices across all the in-app features offered by an app. All the prices are recorded in dollars. The key independent variables for our investigation are Price of the App, Mean In-App Price, and the interaction (Price of the App) X (Mean In-App Price). Since it is right-skewed with a mean that is substantially higher than the median and the mode for all 18 categories, we log-transform the Mean In-App Price as well.

Mobile apps are heterogeneous in terms of their themes (e.g., education, arcade games, etc.) and the number and complexity of attributes [29]. Such differences can affect how consumers choose, use, and assess products $([19,27])$. We, therefore, use multiple variables to capture the role of attributes in the valence and volume of ratings. Specifically, we include the following variables: (1) Size in megabytes, (2) rating of the app's content - a numerical scale with four ratings: $4,9,12$, and 17 where a specific rating indicates that the app is suitable only for individuals who are older than that rating; we used rating 17 as the baseline and represented the other three ratings by three dummy variables Rating4, Rating9, and Rating12, (3) the Number of Languages that the app is available in, (4) the Number of Versions of the app released since its launch, and (5) Compatibility - a variable that represents whether the app is compatible for use on all of Apple's consumer electronics devices (which the App store refers to as universal compatibility) or is meant only for iPhone, or only for iPad. The variable is set to 1 if the app is universally compatible and 0 if it can only be used with the iPhone or iPad. We include this variable to capture any increase in an app's value to consumers due to universal compatibility. (6) To control for the differences in the popularity of the themes, we include one dummy variable for each of the 17 categories of apps in our data with the 18th and smallest category (in terms of the number of apps available for the category), Dice, serving as the baseline. (7) Finally, to control for heterogeneity in experience in attracting consumer ratings, we include a variable that represents the Number of Apps developed and sold by the app's developer.

We also include five additional variables to capture the effects of promotions: (1) the Number of Screenshots of the app on its page in the App store, (2) iPhone Homepage Promotions which is the number of times that the app was promoted on the home page of the iPhone App Store from its first release until the time that we collected our data, (3) iPhone Inside Promotions - analogous count for promotions of the app on inner pages of the App store for iPhone, (4) iPad Homepage Promotions - which is the count of promotions of the app on the home page of the iPad app store since its first release, and (5) iPad Inside Promotionsanalogous measure for promotions of the app on the inner pages of the App store for iPad. Table 1 presents descriptive statistics of the variables for our sample. Tables 2 and 3 provide summaries of the valence and volume of ratings, respectively, for each of the 18 categories of apps in the data. Table 4 provides the average prices of in-app features in each category.

It is clear from these tables that, in addition to the diversity of 18 different categories, the apps in our sample are also highly diverse in their base prices which range from $\$ 0$ to $\$ 19.99$ and the prices of the in-app products which are between $\$ 0$ and $\$ 999.99$. Additionally, the minimum prices of the in-app products which range from $\$ 0$ to $\$ 49.99$ and the maximum prices of the in-app products which are between $\$ 0$ and $\$ 999.99$ are themselves highly diverse. Further, the in-app prices exhibit substantial variation within categories. Finally, the apps also display substantial heterogeneity in the promotional support, sizes, number of versions released, and number of languages that they are available in. We rely on this diversity of the apps in our sample in all the variables used in our empirical investigation as a control for endogeneity. Such diversity reduces the likelihood of systematic correlation between prices and omitted variables. ${ }^{3}$ Some limitations of the data, however, are that it only includes apps sold through a single distributor. Additionally, all the apps are related to various categories of games and do not include other categories such as productivity.

We regress each dependent variable separately on the independent variables to investigate our research questions. Additionally, we include the interaction of Price of the App and Mean In-App Price. We also include the square of Price of the $A p p$ to allow for any non-linear effects of the app's price. Seventy percent of the sample is used for model calibration with the remaining thirty percent retained for holdout testing. Prior to model calibration, we log-transform and standardize the Valence and Volume of ratings and Mean In-App Price. All other continuous variables are standardized.

\footnotetext{
${ }^{3}$ We would like to thank an anonymous reviewer and the Guest Editor for prompting us to include this discussion.
} 
Table 1 Descriptive statistics of variables in the data

\begin{tabular}{|c|c|c|c|c|c|c|}
\hline & Mean & Median & Mode & Minimum & Maximum & Standard deviation \\
\hline Number of screenshots & 18.07 & 21 & 21 & 3 & 21 & 4.46 \\
\hline Number of languages & 3.66 & 1 & 1 & 1 & 38 & 5.43 \\
\hline Size & 66.97 & 40 & 46.6 & 1 & $1,017.1$ & 108.61 \\
\hline Number of apps & 43.55 & 17 & 3 & 1 & 1,573 & 98.21 \\
\hline Number of in-app features & 6.17 & 6 & 10 & 1 & 10 & 3.28 \\
\hline Minimum in-app price & 1.56 & 0.99 & 0.99 & 0 & 49.99 & 1.5 \\
\hline Maximum in-app price & 27.92 & 9.99 & 99.99 & 0 & 999.99 & 38.29 \\
\hline Mean in-app prices & 8.91 & 4.87 & 0.99 & 0 & 192.43 & 9.86 \\
\hline Standard deviation of in-app prices & 8.83 & 3.44 & 0 & 0 & 322.06 & 12.57 \\
\hline iPhone homepage Promotions & 1.78 & 0 & 0 & 0 & 341 & 14.72 \\
\hline iPhone inside promotions & 90.75 & 27 & 0 & 0 & 2,271 & 168.13 \\
\hline iPad homepage promotions & 1.6 & 0 & 0 & 0 & 342 & 13.88 \\
\hline iPad inside promotions & 80.59 & 19 & 0 & 0 & 2,109 & 152.58 \\
\hline Number of versions & 8.61 & 6 & 1 & 1 & 80 & 8.86 \\
\hline Price of the app & 0.45 & 0 & 0 & 0 & 19.99 & 1.2 \\
\hline
\end{tabular}

\section{Results}

We assessed the reliability of the estimates by using them to predict the dependent variables in the holdout sample. Figures 2 and 3 compare the predicted and actual values of the valence and volume of ratings for a random sample of 50 apps drawn from the holdout sample. Both figures suggest that the models are able to follow variations of the dependent variables reasonably well.

Table 5 presents the mean absolute deviation (MAD), mean absolute percentage error (MAPE), and root mean squared error
(RMSE) of the predictions for the valence and volume of ratings. The errors in predicting the valence of ratings are quite low. The errors in predicting the volume, however, are higher perhaps due to the large range $(5$ to 171,435$)$ of this variable.

\subsection{Results from the Valence of Ratings Model}

Estimates of the parameters of the valence model are presented in Table 6. Our first research question was on whether the base price of an app affects the valence of its ratings. The
Table 2 Descriptive statistics of valence of ratings in each category

\begin{tabular}{llllllll}
\hline & N & Mean & Median & Mode & Minimum & Maximum & Standard deviation \\
\hline Action & 646 & 4.32 & 4.4 & 4.6 & 1.80 & 5.00 & 0.45 \\
Adventure & 561 & 4.21 & 4.3 & 4.5 & 1.80 & 5.00 & 0.51 \\
Arcade & 592 & 4.31 & 4.4 & 4.5 & 2.20 & 5.00 & 0.43 \\
Board & 385 & 4.19 & 4.3 & 4.6 & 1.60 & 5.00 & 0.58 \\
Card & 409 & 4.13 & 4.3 & 4.4 & 1.00 & 5.00 & 0.62 \\
Casino & 418 & 4.14 & 4.3 & 4.4 & 1.00 & 5.00 & 0.60 \\
Dice & 282 & 4.17 & 4.3 & 4.4 & 1.90 & 5.00 & 0.56 \\
Education & 452 & 3.93 & 4.0 & 3.9 & 2.10 & 5.00 & 0.53 \\
Family & 576 & 4.07 & 4.2 & 4.4 & 2.00 & 5.00 & 0.53 \\
Music & 397 & 4.00 & 4.2 & 4.4 & 1.00 & 5.00 & 0.72 \\
Puzzle & 568 & 4.31 & 4.4 & 4.5 & 1.80 & 5.00 & 0.49 \\
Racing & 471 & 4.09 & 4.2 & 4.3 & 2.00 & 5.00 & 0.50 \\
Role-playing & 662 & 4.17 & 4.3 & 4.6 & 1.40 & 5.00 & 0.62 \\
Simulation & 601 & 4.19 & 4.3 & 4.4 & 1.60 & 5.00 & 0.52 \\
Sports & 462 & 4.12 & 4.2 & 4.5 & 1.30 & 5.00 & 0.57 \\
Strategy & 567 & 4.22 & 4.4 & 4.6 & 1.60 & 5.00 & 0.58 \\
Trivia & 474 & 4.15 & 4.3 & 4.5 & 1.20 & 5.00 & 0.64 \\
World & 510 & 4.23 & 4.4 & 4.5 & 1.60 & 5.00 & 0.58 \\
\hline
\end{tabular}


Table 3 Descriptive statistics of volume of ratings in each category

\begin{tabular}{lrrrrrrr}
\hline & N & Mean & Median & Mode & Minimum & Maximum & Standard deviation \\
\hline Action & 646 & 1,828 & 204 & 5 & 5 & 171,435 & 8,816 \\
Adventure & 561 & 2,054 & 205 & 5 & 5 & 171,435 & 9,986 \\
Arcade & 592 & 1,299 & 220 & 11 & 5 & 55,650 & 4,085 \\
Board & 385 & 380 & 53 & 9 & 5 & 25,688 & 1,577 \\
Card & 409 & 267 & 49 & 5 & 5 & 12,812 & 921 \\
Casino & 418 & 271 & 36 & 5 & 5 & 26,129 & 1,400 \\
Dice & 282 & 224 & 23 & 11 & 5 & 26,129 & 1,610 \\
Education & 452 & 274 & 57 & 10 & 5 & 33,033 & 1,608 \\
Family & 576 & 648 & 130 & 5 & 5 & 34,753 & 2,032 \\
Music & 397 & 245 & 28 & 6 & 5 & 11,185 & 952 \\
Puzzle & 568 & 1,136 & 100 & 5 & 5 & 43,486 & 3,979 \\
Racing & 471 & 377 & 88 & 5 & 5 & 13,427 & 968 \\
Role-playing & 662 & 786 & 94 & 6 & 5 & 28,086 & 2487 \\
Simulation & 601 & 1,092 & 123 & 57 & 5 & 89,145 & 5,238 \\
Sports & 462 & 929 & 84 & 5 & 5 & 56,649 & 3,729 \\
Strategy & 567 & 642 & 71 & 6 & 5 & 33,071 & 2,536 \\
Trivia & 474 & 496 & 49 & 5 & 5 & 45,224 & 2,499 \\
World & 510 & 458 & 59 & 5 & 5 & 38,001 & 1,956 \\
& & & & & & & \\
& & & 59 & 5 & 5 & 5 & \\
\end{tabular}

estimate for Price of the App indicates that the base price does not have a significant effect on the valence of ratings. Thus, at least in the mobile app category, there is no evidence that the prices of the base products influence the valence of consumer ratings.

Turning to our second question, the estimated effect of Mean In-App Price on the valence of ratings is positive and significant. This suggests that the prices of add-ons have a positive effect on the valence of ratings in the mobile app category. Thus, users who buy in-app features are likely to be those who derive additional utility and value from the app.

Finally, the estimated effect of the interaction of Price of the App and Mean In-App Price is negative and significant. This is in contrast to the main effect of in-app prices which have a positive effect on valence. The significant negative interaction of Mean In-App Price with Price of the App
Table 4 Descriptive statistics of in-app prices in each category

\begin{tabular}{llllllll}
\hline & $N$ & Mean & Median & Mode & Minimum & Maximum & Standard deviation \\
\hline Action & 646 & 11.07 & 6.68 & 0.99 & 0.99 & 45.21 & 10.40 \\
Adventure & 561 & 11.80 & 7.62 & 0.99 & 0.99 & 192.43 & 12.95 \\
Arcade & 592 & 8.27 & 5.31 & 0.99 & 0.99 & 36.56 & 8.35 \\
Board & 385 & 5.19 & 2.29 & 0.99 & 0.99 & 36.99 & 6.45 \\
Card & 409 & 13.00 & 8.49 & 0.99 & 0.99 & 38.89 & 11.12 \\
Casino & 418 & 13.34 & 9.94 & 0.99 & 0.99 & 49.99 & 9.85 \\
Dice & 282 & 10.48 & 6.49 & 0.99 & 0.99 & 49.99 & 10.04 \\
Education & 452 & 3.25 & 2.79 & 1.99 & 0.99 & 31.99 & 2.58 \\
Family & 576 & 5.66 & 3.19 & 0.99 & 0.99 & 34.62 & 6.46 \\
Music & 397 & 6.03 & 2.99 & 0.99 & 0.99 & 42.49 & 6.95 \\
Puzzle & 568 & 6.36 & 2.99 & 0.99 & 0.00 & 37.49 & 7.45 \\
Racing & 471 & 7.21 & 4.19 & 0.99 & 0.99 & 51.09 & 8.03 \\
Role-playing & 662 & 14.44 & 8.99 & 0.99 & 0.99 & 192.43 & 14.02 \\
Simulation & 601 & 9.80 & 5.99 & 0.99 & 0.99 & 51.09 & 9.61 \\
Sports & 462 & 7.72 & 4.27 & 0.99 & 0.99 & 36.19 & 8.68 \\
Strategy & 567 & 12.87 & 7.59 & 0.99 & 0.99 & 50.49 & 12.10 \\
Trivia & 474 & 5.43 & 4.46 & 0.99 & 0.99 & 31.66 & 4.41 \\
World & 510 & 5.72 & 4.49 & 0.99 & 0.99 & 101.69 & 6.17 \\
\hline
\end{tabular}




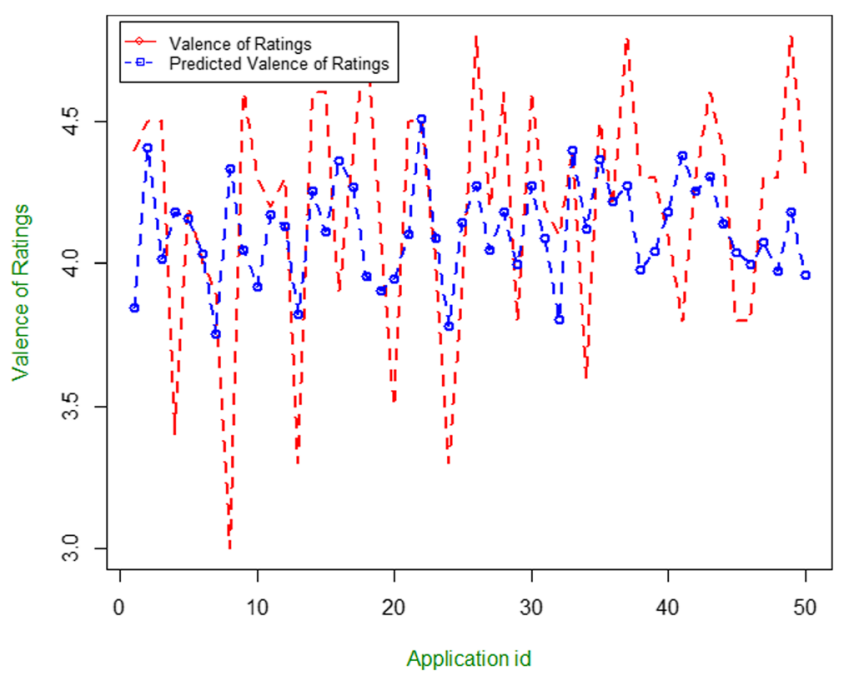

Fig. 2 Predicted and actual valence of ratings

suggests that the addition of in-app prices to the mental account for the app hurts ratings. Specifically, higher totals of app and in-app prices result in lower valence. Our findings in the mobile app category, therefore, suggest that consumers maintain mental accounts of the total expenditures on base products and add-ons.

\subsection{Discussion of Other Effects in the Valence Model}

Turning to the other variables, the Minimum In-App Price has a significant negative effect on the valence of ratings. This suggests that apps that offer multiple in-app features should set a low price for the least expensive feature. The Standard Deviation of In-App Prices also has a negative effect suggesting that firms should lower the variance of the prices of addons to increase the valence of consumer ratings.

In terms of the attributes, the negative estimate of Number of In-App Features is interesting and suggests potential choice

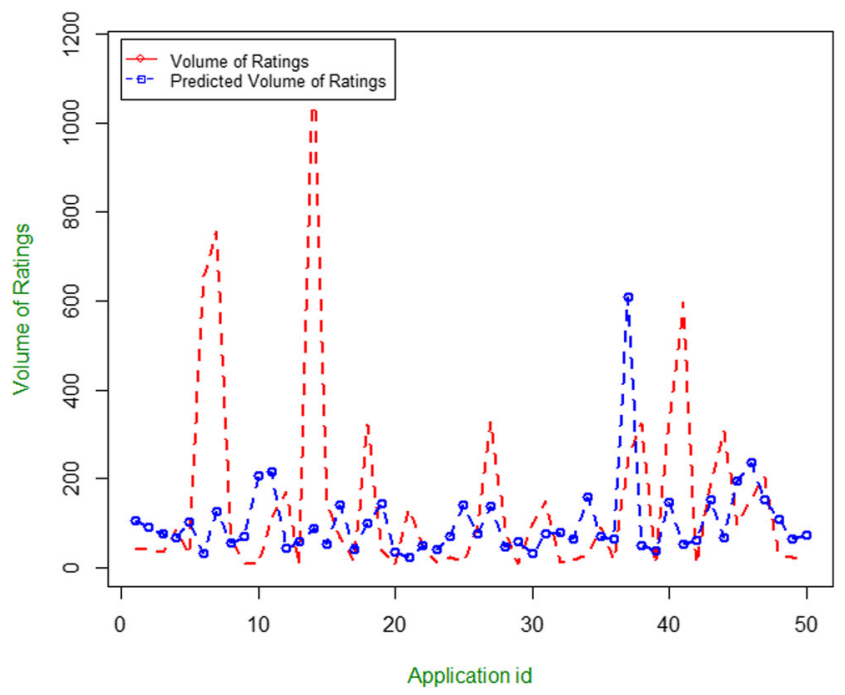

Fig. 3 Predicted and actual volume of ratings
Table 5 Predictive performance of the models for the valence and volume ratings

\begin{tabular}{lll}
\hline & Valence of ratings & Volume of ratings \\
\hline MAD & 0.41 & 787.27 \\
MAPE & 0.11 & 2.97 \\
RMSE & 0.55 & $3,840.01$ \\
\hline
\end{tabular}

overload effects [15]. The estimate for Number of Apps is also negative and suggests that apps from developers with more experience attract less positive ratings. While we do not have a theoretical explanation for this, we believe that this could be due to higher consumer expectations for apps from developers with more experience. Another potential explanation is that firms that offer multiple apps are unable to satisfactorily serve the customers of all the apps. Interestingly, apps that are compatible with multiple products, rather than with just one such as the iPhone or the iPad, are also likely to be rated lower than those that can be used with a single product.

Turning to promotions, the Number of Screenshots increases the valence of ratings. Finally, most of the category effects are not significant. The exceptions are the Puzzle category which has a significant positive effect and Education and Music categories both of which have negative effects.

\subsection{Results from the Volume of Ratings Model}

Table 7 has the estimated parameters of the volume of ratings model. The estimated effect of Price of the App is negative and significant but that of Squared Price of the App is positive and significant. This non-linear relationship suggests that the role of base price in the volume of ratings varies. Specifically, below a threshold, it plays an allocative role and reduces volume but switches to an informative role above the threshold and has a positive effect on the volume of ratings. The estimated net effect of Mean In-App Price on the volume of ratings is positive and significant. In light of the positive effect of this variable on the valence of ratings as well, this suggests that in-app purchases are likely to be made by users who use the app and seek additional utility. This increase in utility increases the app's value and increases the volume of ratings.

\subsection{Discussion of Other Effects in the Volume Model}

Minimum In-App Price and Standard Deviation of In-App Prices have a significant negative effect on the volume of ratings. As in the case of valence, apps that offer multiple in-app features should therefore consider setting a low price for the least expensive feature, and should consider lowering the variance of the prices for the add-ons, in order to increase the volume of ratings.

With regard to the attributes, an increase in the Number of In-App Features increases the volume of ratings perhaps 
Table 6 Estimated parameters of the valence of ratings model

\begin{tabular}{|c|c|c|c|c|c|c|c|}
\hline & Estimate & Standard error & $t$ value & & Estimate & Standard error & $t$ value \\
\hline (Intercept) & $0.2961 *$ & 0.1023 & 2.8940 & Categories & & & \\
\hline Price variables & & & & Action & 0.1289 & 0.0860 & 1.4990 \\
\hline Price of the app & 0.0021 & 0.0206 & 0.1010 & Adventure & -0.0176 & 0.0865 & -0.2040 \\
\hline Mean in-app price & $0.1776^{*}$ & 0.0255 & 6.9750 & Arcade & 0.1654 & 0.0858 & 1.9270 \\
\hline (Price of the app) X (Mean in-app price) & $-0.0352 *$ & 0.0125 & -2.8220 & Board & 0.1604 & 0.0921 & 1.7410 \\
\hline Minimum in-app price & $-0.0774 *$ & 0.0141 & -5.5050 & Card & -0.1536 & 0.0904 & -1.7000 \\
\hline Standard deviation of in-app price & $-0.1021^{*}$ & 0.0231 & -4.4160 & Casino & -0.0995 & 0.0918 & -1.0840 \\
\hline Squared price of the app & 0.0015 & 0.0029 & 0.5370 & Education & $-0.2631^{*}$ & 0.0911 & -2.8890 \\
\hline Attributes of app & & & & Family & -0.1328 & 0.0872 & -1.5230 \\
\hline Size & 0.0166 & 0.0139 & 1.1940 & Music & $-0.2093^{*}$ & 0.0914 & -2.2900 \\
\hline Number of in-app features & $-0.0363^{*}$ & 0.0150 & -2.4210 & Puzzle & $0.2494^{*}$ & 0.0876 & 2.8460 \\
\hline Rating4 & -0.0112 & 0.0603 & -0.1850 & Racing & -0.1387 & 0.0895 & -1.5500 \\
\hline Rating9 & 0.0433 & 0.0666 & 0.6500 & Role-playing & -0.1155 & 0.0838 & -1.3790 \\
\hline Rating12 & 0.0150 & 0.0632 & 0.2380 & Simulation & -0.0631 & 0.0858 & -0.7360 \\
\hline Number of languages & -0.0114 & 0.0125 & -0.9140 & Sports & -0.1232 & 0.0898 & -1.3730 \\
\hline Number of apps & $-0.0403 *$ & 0.0129 & -3.1170 & Strategy & 0.0295 & 0.0868 & 0.3390 \\
\hline Compatibility & $-0.3386^{*}$ & 0.0554 & -6.1150 & Trivia & -0.0521 & 0.0897 & -0.5810 \\
\hline Number of versions & $0.0462 *$ & 0.0134 & 3.4420 & World & 0.1067 & 0.0877 & 1.2170 \\
\hline Promotions & & & & $R^{2}$ & 0.068 & & \\
\hline Number of screenshots & $0.1851^{*}$ & 0.0202 & 9.1550 & & & & \\
\hline iPhone homepage promotions & 0.0048 & 0.0304 & 0.1560 & & & & \\
\hline iPhone inside promotions & -0.0504 & 0.0355 & -1.4190 & & & & \\
\hline iPad homepage promotions & -0.0427 & 0.0308 & -1.3870 & & & & \\
\hline iPad homepage promotions & $0.1459^{*}$ & 0.0365 & 3.9960 & & & & \\
\hline
\end{tabular}

*Significant at 0.05 or lower

because there are more features that users can evaluate. Nonetheless, given the finding that increasing the number of in-app features hurts valence, the increase in volume could be due to ratings of lower valence. Firms should, therefore, not offer a large number of in-app features. The Size of the app has a positive effect suggesting that larger apps attract more ratings. The Number of Apps developed and sold by the firm also has a positive effect on volume. The estimates also suggest that apps that are rated for users that are 12 years of age or older are more likely to attract ratings than others. Compatibility and Number of Versions, on the other hand, have negative effects suggesting that apps that are universally compatible or changed often attract fewer ratings than those that can only be used on iPad and iPhone or are updated less frequently. This is similar to the negative effect of these variables on valence.

The only promotion that has a significant positive effect on the volume of ratings for an app is that of iPhone Inside Promotions. Finally, all significant category effects are positive relative to the baseline. The estimated effects of the categories, presented in order of decreasing magnitudes, suggest that action, adventure, arcade, simulation, and family have the most positive effects.

\section{Discussion}

Our findings identify significant interactions between the base price and the prices of the add-ons in the base + add-on pricing model. Specifically, we find a negative interaction between the two prices for the valence of ratings but a positive interaction for the volume of ratings. What are the implications of these findings for the pricing of base products and add-ons in extremely competitive categories to manage the valence and volume of consumer ratings? We discuss this next.

\subsection{Pricing Implications}

We consider two levels of base and add-on prices in the app category: low (LB) and high (HB) base prices corresponding to the least and highest Price of the App, respectively, in our sample, and low (LA) and high (HA) add-on prices corresponding to the least and highest Mean In-App Price, respectively, in the sample. We then use the estimates from the models to predict the valence and volume of ratings for the (LB,LA), (LB,HA), (HB,LA), and (HB,HA) combinations

The predictions from the two models presented in Figs. 4 and 5 provide insights into the interactive effects of the two 
Table 7 Estimated parameters of the volume of ratings model

\begin{tabular}{|c|c|c|c|c|c|c|c|}
\hline & Estimate & Standard error & $t$ value & & Estimate & Standard error & $t$ value \\
\hline (Intercept) & $-0.4075^{*}$ & 0.0991 & -4.1110 & Categories & & & \\
\hline Price variables & & & & Action & $0.9352 *$ & 0.0833 & 11.2220 \\
\hline Price of the app & $-0.0510^{*}$ & 0.0199 & -2.5570 & Adventure & $0.9322 *$ & 0.0839 & 11.1170 \\
\hline Mean in-app price & $0.1167 *$ & 0.0247 & 4.7320 & Arcade & $0.9277^{*}$ & 0.0832 & 11.1510 \\
\hline (Price of the app) $x$ (Mean in-app price) & 0.0213 & 0.0121 & 1.7600 & Simulation & $0.7649 *$ & 0.0831 & 9.2010 \\
\hline Minimum in-app price & $-0.0712 *$ & 0.0136 & -5.2260 & Family & $0.7141 *$ & 0.0845 & 8.4500 \\
\hline Standard deviation of in-app price & $-0.0897^{*}$ & 0.0224 & -4.0040 & Puzzle & $0.6803^{*}$ & 0.0849 & 8.0100 \\
\hline Squared price of the app & $0.0084 *$ & 0.0028 & 3.0430 & Roleplaying & $0.6438^{*}$ & 0.0812 & 7.9300 \\
\hline Attributes of app & & & & Sports & $0.5855^{*}$ & 0.087 & 6.7320 \\
\hline Size & $0.0347^{*}$ & 0.0135 & 2.5690 & Racing & $0.5087^{*}$ & 0.0868 & 5.8650 \\
\hline Number of in-app features & $0.0404 *$ & 0.0145 & 2.7830 & Trivia & $0.4574 *$ & 0.0869 & 5.2610 \\
\hline Rating4 & -0.0419 & 0.0584 & -0.7170 & Strategy & $0.4563^{*}$ & 0.0841 & 5.4240 \\
\hline Rating9 & -0.0465 & 0.0645 & -0.7210 & Board & $0.4482 *$ & 0.0893 & 5.0210 \\
\hline Rating12 & $0.0088 *$ & 0.0612 & 0.1440 & World & $0.4349 *$ & 0.0849 & 5.1200 \\
\hline Number of languages & $-0.0360 *$ & 0.0121 & -2.9710 & Education & $0.4303 *$ & 0.0883 & 4.8760 \\
\hline Number of apps & $0.0347^{*}$ & 0.0125 & 2.7660 & Card & $0.2763 *$ & 0.0876 & 3.1540 \\
\hline Compatibility & $-0.1747^{*}$ & 0.0537 & -3.2560 & Casino & $0.1862 *$ & 0.0889 & 2.0940 \\
\hline Number of versions & $-0.0671^{*}$ & 0.0130 & -5.1570 & Music & $0.1852 *$ & 0.0886 & 2.0910 \\
\hline Promotions & & & & $R^{2}$ & 0.1248 & & \\
\hline Number of screenshots & 0.0321 & 0.0196 & 1.6360 & & & & \\
\hline iPhone homepage promotions & -0.0166 & 0.0295 & -0.5620 & & & & \\
\hline iPhone inside promotions & $0.1721 *$ & 0.0344 & 5.0010 & & & & \\
\hline iPad homepage promotions & -0.0181 & 0.0298 & -0.6060 & & & & \\
\hline iPad inside promotions & 0.0146 & 0.0354 & 0.4130 & & & & \\
\hline
\end{tabular}

* Significant at 0.05 or lower

prices on the valence and volume of ratings and identify desirable combinations. The predicted valence of ratings suggests that it will be highest under the high app price and low in-app price combination. The combination of a low app price and low in-app prices on the other hand results in the lowest valence and is, clearly, the least desirable. A combination of high app and high in-app prices results in the second-lowest valence. On the other hand, the low app and high in-app price combination leads to the second-highest valence.

The predicted volumes of ratings in Fig. 5 suggest that the combination of high app and high in-app prices leads to the largest volume of ratings. Taken together with the predicted second lowest valence under this condition, however, this means that the volume of low valence ratings could be very high for this price combination. Interestingly, the high appprice and Low in-app price combination which leads to the highest valence results in the lowest volume of ratings. The most attractive combination would, therefore, be low app price and high in-app prices which leads to the second highest volume of ratings while also producing the second-highest valence. Our key finding, therefore, is that the classic low base + high add-on pricing model is the best option to generate high volumes of positive word of mouth from consumers. Firms, therefore, should not rely on lowering both base and add-on prices to stimulate consumer ratings even in extremely competitive categories.

\subsection{Benefits and Costs of Moving Between Price Combinations}

Firms competing in the app market have few formal insights into pricing their products. For instance, one of the two largest distributors of mobile applications, the Google Play store, offers guidelines such as: "Your apps may be able to bear a higher price in some markets" [2]. Given the absence of formal insights or guidelines regarding pricing, firms appear to set prices based on what they believe they might be able to charge rather than what prices might increase consumer response as well as the valence and volume of ratings. The desirability of the low app price and high in-app prices combination suggested by our findings, thus, raises a managerially important question. What are the costs and benefits of a specific combination of app and in-app feature prices being used by developers? Specifically, what is the cost or benefit in terms of valence and volume of ratings for apps using a specific combination of prices when they switch to a different 
Fig. 4 Predicted valence of ratings in four base + add-on pricing scenarios. $L B$ low base price, $H B$ high base price, $L A$ low in-app prices, $H A$ high in-app prices

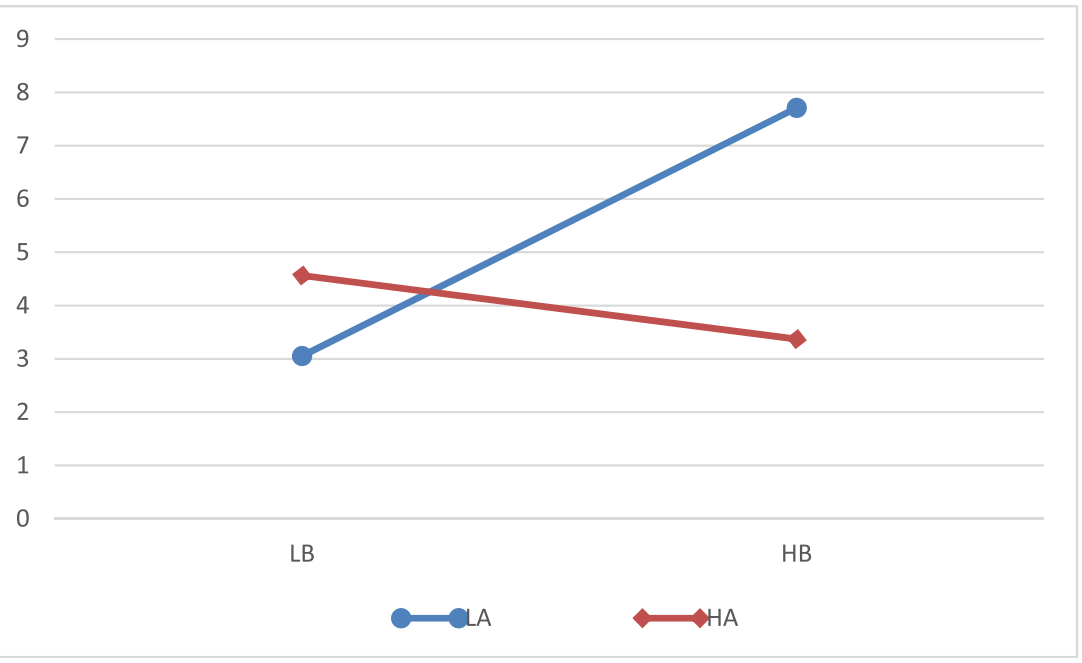

combination such as that of low app-price and high in-app prices identified as the best by our results? We explore this through a simulation of the effects of changing from the currently used combination of prices to a different combination for apps in our data. Specifically, we consider four groups of apps where each group follows one of the four combinations (LB,LA), (LB,HA), (HB,LA), and (HB,HA). For each group, we use the estimated valence and volume of ratings models to simulate the effect of switching from the combination it uses to each of the other three combinations (details of the simulation are included in the Appendix).

Table 8 presents the results of the simulation. Entries along the diagonal of the table provide the actual average valence of ratings and actual average volume of ratings for each combination. These entries confirm the two key findings from the empirical analysis: the (LB,LA) combination has the lowest average valence of ratings and the (HB,HA) combination has the highest average volume of ratings. Thus, despite their low explanatory power in terms of $R^{2}$, the models are able to capture the true patterns in the data well.
The off-diagonal entries present the likely valence and volume of ratings if apps in a group were to move to a different pricing combination. For instance, the off-diagonal entries in the column corresponding to the (LB,HA) combination, provide insights into the benefits and costs for apps moving to this combination from other pricing combinations. These entries provide a clear indication that moving to this combination of prices from the (LB,LA) or (HB,LA) combinations is beneficial either in terms of higher valence or volume of ratings generated. For example, switching to this combination from the (LB,LA) combination results in higher valence of ratings though the volume of ratings is lower. The same is the case for a switch from the (HB,LA) combination to (LB,HA).

In contrast, however, switching into the (HB,LA) combination is beneficial for some combinations and not so for others. For instance, apps following (LB,LA) gain from the move both in the valence and volume of ratings. Those using the (LB,HA) combination, however, would face substantial losses in the volume of ratings.
Fig. 5 Predicted volume of ratings (standardized) in four base + add-on pricing scenarios. $L B$ low base price, $H B$ high base price, $L A$ low in-app prices, $H A$ high in-app prices

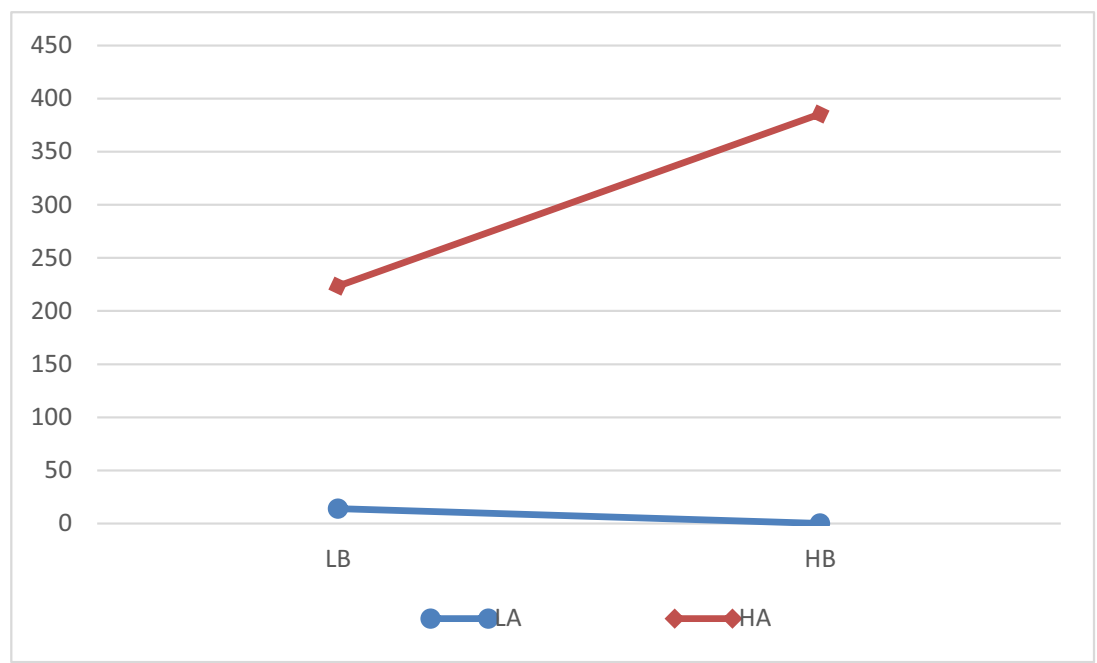


Table 8 Simulation analysis of effects of changing price combinations on valence and volume of ratings

\begin{tabular}{lllll}
\hline & $(\mathrm{LB}, \mathrm{LA})$ & $(\mathrm{LB}, \mathrm{HA})$ & $(\mathrm{HB}, \mathrm{LA})$ & $(\mathrm{HB}, \mathrm{HA})$ \\
\hline (LB,LA) & 4.08 & 4.55 & 4.45 & 3.61 \\
& 538 & 266 & 2,413 & 3,0484 \\
$(\mathrm{LB}, \mathrm{HA})$ & 3.11 & 4.23 & 8.19 & 4.42 \\
& 16 & 939 & 0 & 2,351 \\
(HB,LA) & 4.16 & 4.60 & 4.10 & 4.57 \\
& 148 & 290 & 555 & 327 \\
(HB,HA) & 3.05 & 4.12 & 3.12 & 4.21 \\
& 19 & 146 & 17 & 1,117 \\
\hline
\end{tabular}

Note: Entries in each cell represent average valence and volume of ratings

Entries in the (HB,HA) column demonstrate the costs and benefits of moving to this combination from other combinations. For instance, switching from (LB,LA) pricing leads to a drop in valence to 3.61 and a substantial increase in the volume of ratings. Thus, moving to this combination could be very risky for apps following the (LB,LA) pricing model. In contrast, however, apps following either the (LB,HA) or (HB,LA) combinations would gain by switching to the (HB,HA) combination of prices in the valence and/or the volume of ratings.

Overall, the results of the simulation suggest that the (low base, high add-on) pricing model is the most attractive option for apps in terms of the valence of ratings. This is followed by the (high base, low add-on) combination which also results in an increase in the valence or volume of ratings. The (high base, high add-on) combination is also an attractive pricing model since it not only results in higher ratings but also in substantially higher volume of ratings in most cases. The exception, however, is in the case of switching from (LB,LA) which leads to a substantial increase in the volume of ratings of lower valence. The least attractive combination, however, is (LB,LA) switching to which results in lower valence, lower volume, or both for the other three combinations. Thus, the results of the simulation provide additional support to the implications from the plots in Figs. 4 and 5.

\subsection{Future Research}

Our research focuses on identifying desirable combinations of prices in the base + add-on pricing model in extremely competitive categories. Our empirical results suggest that prices in these markets play both an informative and an allocative role and are also subject to mental accounting. They also suggest that the most desirable combination of base and add-on prices is a low price of the base and higher prices of the add-ons. Interestingly, the least attractive option is a (low base, low add-on) combination despite the extreme competition. This combination results in the lowest valence of ratings. This is perhaps one of the more surprising findings of our investigation since many firms in the mobile app category rely on this combination of prices.

Our results, however, are limited to a single category. The number and variety of markets that are extremely competitive is quite large. Additional research on the generalizability of our findings through replications in markets such as freeware, online music, online radio services, and subscription blogs is therefore important. Results from such investigations can help in further improving the base + add-on pricing model in extremely competitive categories.

Our results also suggest that a high minimum price of the in-app features suppresses both the valence and volume of ratings. An important question for investigation from theoretical and practical perspectives, therefore, is why this is so in light of our other finding that higher averages of in-app prices have a positive effect. One explanation for this pattern could be that users who buy in-app features - the add-ons - react negatively to buying the first in-app feature at a high price but that, once they start using them, the prices of in-app features become less salient. Empirical investigations regarding whether this is the case can provide valuable insights into the pricing of add-on features in extremely competitive categories.

Another possible extension is on how the potential twosided nature of the mobile-app market plays a role in pricing by firms selling apps through large platforms like the App Store. As per current practices, these platforms neither charge a fee for listing apps nor play a role in how firms price their apps. The traditional issues in pricing in two-sided markets, such as price discrimination and the platforms' roles in pricing, are therefore not currently relevant for the questions that we investigate in this research. They may, however, become relevant in future if the pricing policies of the platforms change requiring firms to pay an upfront fee to participate in the market.

\section{Appendix}

Simulation of the effects of changing the combination of price and average in-app price on the valence and volume of ratings

We consider four combinations of app-price and in-app prices:

\begin{tabular}{lll}
\hline App price & Average in-app price & \\
& Low (LA) & High (HA) \\
Low (LB) & (LB,LA) & (LB,HA) \\
High (HB) & (HB,LA) & (HB,HA) \\
\hline
\end{tabular}

Empirically, we define each of the four pricing options LB, $\mathrm{HB}, \mathrm{LA}$, and HA as follows: 
- $\quad \mathrm{LB}=$ price of the app is in the first quartile of the distribution of app prices in the data

- $\mathrm{HB}=$ price of the app is in the fourth quartile of the distribution of app prices in the data

- $\quad \mathrm{LA}=$ average in-app price for the app is in the first quartile of the distribution of average in-app prices in the data

- $\mathrm{HA}=$ average in-price for the app is in the fourth quartile of the distribution of average in-app prices in the data

We use the estimated model parameters to simulate the effects of changing from one combination of pricing to a different combination using the following steps:

1. Assign the apps in the sample to one of the four pricing combinations (LB,LA), (LB,HA), (HB,LA), and (HB, $\mathrm{HA})$ based on their observed pricing behavior.

2. Consider the (LB,LA) combination:

a. Compute the observed average valence and volume of ratings for the apps assigned to (LB,LA)

b. Change the price of each app assigned to (LB,LA) to the average price and average in-app prices in the (LB,HA) combination

c. Use the valence and volume of ratings models to recompute the average valence and average volume of ratings of the apps in the (LB,LA) combination

d. Repeat steps (b) and (c) twice by changing the prices of the apps in the (LB,LA) combination to the average price and average in-app prices in the (HB,LA) and (HB,HA) combinations

3. Modify step (2) appropriately and repeat for each of the other three combinations (LB,HA), (HB,LA), and (HB, HA)

\section{References}

1. Anderson EW (1998) Customer satisfaction and word of mouth. J Serv Res 1(1):5-17

2. Android (2014) "Expand into new markets", https://developer. android.com/distribute/users/expand-to-new-markets. html\#localize-your-google-play-listing

3. Arndt J (1967) Role of product-related conversations in the diffusion of a new product. J Mark Res 4:291-295

4. Becker GS (1965) A theory of the allocation of time. Econ J 75: 493-517

5. Bertini M, Wathieu L, Iyengar SS (2012) The discriminating consumer: product proliferation and willingness to pay for quality. J Mark Res 49(1):39-49

6. Dellarocas C, Zhang X, Awad NF (2007) Exploring the value of online product reviews in forecasting sales: the case of motion pictures. J Interact Mark 21(4 Fall):23-45

7. Dichter E (1966) How word-of-mouth advertising works. Harv Bus Rev 44:147-166
8. Dodds WB, Monroe KB, Dhruv Grewal D (1991) Effects of price, brand and store information on buyers' product evaluations. J Mark Res 28(August):307-319

9. Erickson GM, Johansson JK (1985) The role of price in multiattribute product evaluations. J Consum Res 12(2): 195-199

10. Forbes (2013) "Paid apps having a bad time, almost all revenue is from in app purchases in free apps," December 30

11. Fornell C, Johnson MD, Anderson EW, Cha J, Bryant B (1996) The American customer satisfaction index: description, findings, and implications. J Mark 60(4):7-18

12. Friedman, Milton (1957) A theory of the consumption function, Princeton University Press

13. Gabor A, Granger CWJ (1966) Price as an indicator of quality: report on an inquiry. Economica 33:43-70

14. Huang J-H, Chen Y-F (2006) Herding in online product choice". Psychol Mark 23(5):413-428

15. Iyengar SS, Lepper MR (2000) When choice is demotivating can one desire too much of a good thing? J Personal Soc Psychol 79(6): 191-198

16. Kahneman D, Tversky A (1979) Prospect theory: an analysis of decision under risk. Econometrica 47(2):263-292

17. Lalwani AK, Shavitt S (2013) You get what you pay for? Selfconstrual influences price-quality judgments. J Consum Res 40(2):255-267

18. Li X, Hitt LM (2010) Price effects in online product reviews: an analytical model and empirical analysis. MIS Q 34(4):809-831

19. Luce MF, Bettman JR, Payne JW (2001) Tradeoff difficulty: determinants and consequences for consumer decisions. Monogr J Consum Res 1:1-209

20. Noble PM, Gruca TS (1999) Industrial pricing: theory and managerial practice. Mark Sci 18:435-454

21. Oliver RL (1977) Effect of expectation and disconfirmation on post-exposure product evaluations: an alternative interpretation. J Appl Psychol 62:480-86

22. Olshavsky RW, Miller JA (1972) Consumer expectations, product performance and perceived product quality. J Mark Res 9(February):19-21

23. Rao VR (1984) Pricing research in marketing: the state of the art. J Bus 57(1):S39-S60, Part 2: pricing strategy

24. Rao AR, Monroe KB (1989) The effect of price, brand name, and store name on buyers' perceptions of product quality: an integrative review. J Mark Res 26(3):351-57

25. Richins ML. (1984) Word of mouth communication as negative information. In: Advances in Consumer Research, 11, edited by Thomas C. Kinnear, Provo, UT : Association for Consumer Research, 697-702

26. Spreng RA, MacKenzie SB, Olshavsky RW (1996) A reexamination of the determinants of consumer satisfaction. J Mark 60(3):1532 , July

27. Swaminathan V (2003) The impact of recommendation agents on consumer evaluation and choice: the moderating role of category risk, product complexity, and consumer knowledge. J Consum Psychol 13(1):93-101

28. Thaler R (1985) Mental accounting and consumer choice. Mark Sci 4(1985):199-214

29. The Wall Street Journal (2013) Apps rocket toward $\$ 25$ billion in sales, March 4

30. Tripsas M, Gavetti G (2000) Capabilities, cognition, and inertia: evidence from digital imaging. Strat Manag J 21: $1147-1161$

31. Voss GB, Parasuraman A, Grewal D (1998) The role of price and quality perceptions in prepurchase and postpurchase evaluation of services. J Mark 62(October):46-61 
32. Wathieu L, Bertini M (2007) Price as a stimulus to think: the case for willful overpricing. Mark Sci 26(1):118-129

33. Yang Z, Peterson RT (2004) Customer perceived value, satisfaction, and loyalty: the role of switching costs". Psychol Mark 21(10): $799-822$
34. Zeithaml VA (1988) Consumer perceptions of price, quality and value: a means-end model and synthesis of evidence. J Mark 52:2-22

35. Zhu F, Zhang XM (2010) Impact of online consumer reviews on sales: the moderating role of product and consumer characteristics. $\mathrm{J}$ Mark 74(2):133-148 